\title{
Game Design and Learning Objectives for Undergraduate Engineering Ther- modynamics
}

\section{Prof. John M. Pfotenhauer, University of Wisconsin, Madison}

Professor John M. Pfotenhauer earned his BA, MA, and PhD degrees in physics from St. Olaf College and the University of Oregon in 1979, 1981, and 1984. For eight years he conducted research as part of the Applied Superconductivity Center at the University of Wisconsin - Madison before joining the faculty there in the Departments of Mechanical Engineering, and Engineering Physics in 1993. In addition to his research in cryogenics, and in educational games, he teaches courses in thermodynamics, heat transfer, energy systems laboratory, cryogenics, and vacuum technology.

\section{Mr. David J Gagnon, University of Wisconsin - Madison}

David J. Gagnon (University of Wisconsin, Madison) is a Discovery Fellow and program director of the Mobile Learning Lab in the Wisconsin Institutes for Discovery at University of Wisconsin, Madison. He directs a team of educational researchers, software engineers, artists and storytellers that explore the intersections of learning science and media design, specializing in mobile media, video games and simulation. David is also the Director of the ARIS project, a free and open tool that allows anyone to produce mobile games, stories and tours. He is also active member of the Games, Learning and Society Research community.

\section{Mr. Michael Litzkow, University of Wisconsin-Madison}

Mike Litzkow has been a professional software developer on a wide variety of research projects at the University of Wisconsin-Madison since 1983. He has worked on projects ranging from grid computing systems, to parallel architecture simulators, to delivery of course lectures and instructional content over the world wide web. Presently he manages an open-source learning management system for his campus. He participates in the development of games to teach complex technical subjects as time allows.

\section{Dr. Christine Maidl Pribbenow, Wisconsin Center for Education Research}

Dr. Christine M. Pribbenow is the Director of the LEAD Center at UW-Madison. The LEAD Center advances the quality of teaching and learning by evaluating the effectiveness and impact of educational innovations, policies, and practices within higher education. Dr. Pribbenow has degrees in Psychology and Sociology from Carroll University (BS, 1989), Counseling and Student Development in Higher Education from Northern Illinois University (MSEd, 1992), and Higher Education Leadership and Policy Analysis from UW-Madison (PhD, 2000). For the past fifteen years, she has conducted program evaluation for a number of federally funded programs that are designed to improve undergraduate learning, and to increase the representation of women, racial/ethnic minorities, and people with disabilities in STEM. 


\title{
Game Design and Learning Objectives for Undergraduate Engineering Thermodynamics
}

\begin{abstract}
As a gateway course for undergraduate mechanical engineering students, thermodynamics presents a significant challenge for many students. An on-line and deviceaccessible game being developed intends to increase the success rate of students in their introductory thermodynamics course by enabling them to visually interact with the thermodynamic properties of water on the 3D (P-V-T) surface defined by the equation of state. At the entry level, the game explores property relationships in the subcooled-liquid, superheated vapor, and two-phase regions. At the intermediate and higher levels, it challenges players to solve thermodynamics-related professional practice tasks.
\end{abstract}

We report here the results of the first two years of game development, feedback gathered in betatesting sessions, its in-class application, the associated evaluation procedures (Concept Inventory Measurement, student interviews, and game- generated data), and the subsequent re-direction of the game's approach.

As developed in its initial version, the game incorporated the first law energy balance relating work, heat, and internal energy. The game's primary mechanism, although a captivating challenge for its game mechanics, was not configured to address many of the key pedagogical goals associated with the introduction of thermodynamic properties, their inter-dependency, and the unique features of the properties in the subcooled, two-phase, and superheated regions. A relatively cool reaction to the game by the students was reflected in all three evaluation methods and resulted in a significant re-direction of the game's features.

Along with a list of specific pedagogical goals, the game's re-direction includes a set of professional practice scenarios, and a completely new set of game mechanisms. Additional game features, including a novel in-game assessment tool that is based on a combination of Bayesian Knowledge Tracking and Performance Factor Analyses approaches, are also briefly described.

\section{Introduction}

Games can be effective learning tools in classroom settings. This fact has been demonstrated in a wide variety of disciplines, across a large range of ages, and over a long period of time. Indeed, successful examples of 'computerized' games used in university settings may be identified as far back as the 1960's when computers were still in their infant stages. For example, Raia ${ }^{1}$ describes the effective utilization of a computerized game to teach business management skills at the University of Maryland in 1966.

A consistent plea from industries over decades has been the need for universities to train students to handle the complexities and ambiguities of real life problems; to be able to problem solve in complex situations. Chapman and Sorge ${ }^{2}$ contrast classroom learning with real-life situations and argue the need for students to develop skills in communication, critical thinking, and problem solving in order to handle real-life ambiguities. With the significant power of today's computational models, simulations can accurately represent many real-life situations, and these provide access to professional-practice learning opportunities within the constraints of a 
classroom. Spires et al. ${ }^{3}$ note that games can be powerful learning tools because they "can simulate real-world complexity and fast-paced processing in ways that traditional school learning scenarios cannot approximate." Noting that one of the most promising aspects of game learning is their capacity to promote problem-solving skills, Spires et al. ${ }^{3}$ cite the conclusions of the Partnership for $21^{\text {st }}$ Century Skills ${ }^{4}$ that "the capacity to problem solve separates students who are prepared for the increasingly complex life and work environments from those who are not," and those of Levy and Murnane 5 that "the nation's challenge [is] to prepare youth for the highwage/high-skilled jobs that involve expert problem solving skills and complex communication."

Games can also provide valuable experience for students in introductory courses. As McCarthy ${ }^{6}$ notes, students in such courses meet a deluge of terms and definitions within the early weeks of introductory courses. The experience that games afford to repeatedly utilize and relate the many terms within a contextually appropriate environment enables students to quickly solidify the meanings and use of the terms.

In spite of the potential benefits of game-based learning, there are crucial factors that must be included in the design of an educational game in order to realize these benefits. Staalduinen \& Freitas ${ }^{7}$ summarize the recommendations for educational game design from a variety of educational game developers and pedagogical experts, and provide a structural framework for design. Condensing down many helpful features, they define three steps of game design: learning, instruction, and assessment. In the first step game designers need to define learning objectives, clear player goals, and content. In the instruction step they give attention to the learning cycle including user action, feedback (and reflection), engagement, and user learning. In the final step, assessment designers need to include features for debriefing and overall score.

Here we provide a progress report regarding a game being developed by an interdisciplinary team to teach core ideas encountered by undergraduate mechanical engineering students in their introductory course on thermodynamics. Thermodynamics represents one of the more challenging courses for undergraduates in mechanical engineering, and indeed, presents students with a deluge of terms and definitions within the first few weeks. A key objective of the thermodynamics game is therefore to increase the success rate for students in the course by providing a visually interactive space in which they can explore the numerous inter-related parameters and eventually utilize them within a variety of professional practice scenarios.

The game was developed through a sequence of steps that included storyboarding, constructing a simulator, adding elements of playfulness and control, beta-testing, and fine-tuning a variety of features. These led up to a well-designed game, along with a set of carefully developed assessment tools and an external evaluator, for use in a semester based thermodynamics course. Shortly before utilizing the game in class, the development team recognized a mis-match between the game's educational focus and the specific course objectives, and consequently communicated relatively relaxed requirements to the students regarding their game play. The results of the measurements confirmed that indeed the game was not well aligned with the course learning objectives and consequently added minimal value to the learning process. Subsequent redirection of the game is underway so that its features are closely aligned with a detailed set of the course's learning objectives, and naturally lead into the professional practice scenarios. The following sections of this report describe the details of the thermodynamic simulator with its gaming features, its application within the course setting, the associated assessment tools, and its 
subsequent redirected development.

\section{Game Development}

Weekly brainstorming sessions of a cross-disciplinary team featuring expertise in educational game development, web-based programing, educational assessment, and thermodynamics guided the iterative process of construction, testing, and revision that produced the "thermo-game." The team used many of the same processes utilized in their development of the game "Coolit" that teaches principles of cryogenic engineering. At various points in development, colleagues and small groups of students (8-10) informally tested the game and provided helpful feedback resulting in minor modifications of some game features. The following paragraphs describe the details of the core simulator and surrounding game features.

Simulator: In order to help students visualize the inter-relation between thermodynamic properties, the thermo-game provides an interactive 3D space where locations are defined by the three variables, pressure $(\mathrm{P})$, temperature $(\mathrm{T})$, and specific volume $(\mathrm{v})$. The thermodynamic equation of state (EOS) relating these three variables defines a surface within the $3 \mathrm{D}$ space representing all the possible locations, or thermodynamic states, at which a substance can exist. In our game, water is the chosen substance, and the simulator is built around the most recent EOS for water, developed by Wagner and Pruss ${ }^{9}$. The values for all other thermodynamic properties, such as specific enthalpy (h), specific entropy (s), and specific internal energy (u), can be determined at each location, or state. Figure 1 displays the 3D world presented by the initial version of the thermo-game- "thermo-golf."

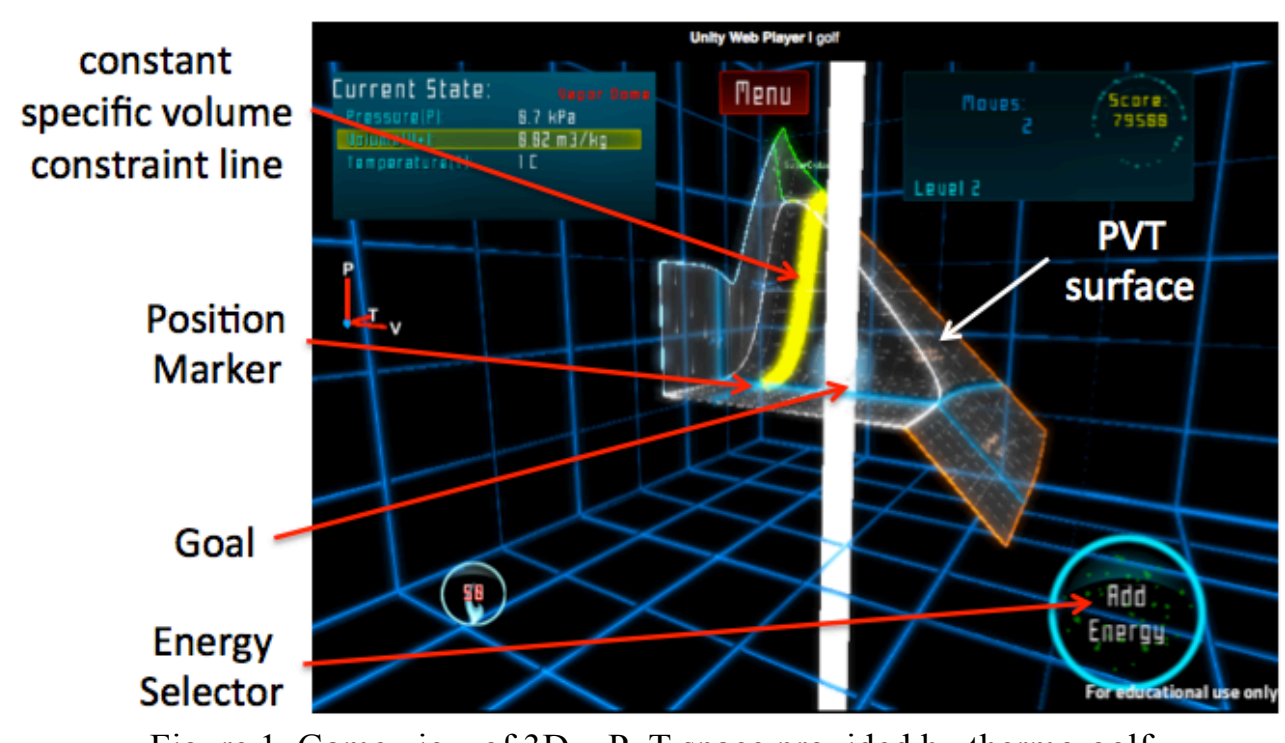

Figure 1. Game view of 3D - PvT space provided by thermo-golf

The EOS provided by Wagner \& Pruss $^{9}$ is valid for the fluid water and extends from the melting curve, to temperatures up to $1273 \mathrm{~K}$ and pressures up to $1000 \mathrm{MPa}$. This range enables exploration into the sub-cooled liquid, superheated vapor, two-phase (liquid-vapor), and critical regions. Properties for the solid phase are, however, not available. Because all of the properties vary over many orders of magnitude within this range, the $\mathrm{P}, \mathrm{T}$, and $\mathrm{v}$ axes are displayed in 
decade, or logarithmic, scales. Interacting with logarithmic changes in these parameters provides an interesting experience for players new to such a large range of scale.

For the purpose of building a game, and for teaching general inter-relationships between the thermodynamic parameters, one may wonder whether 'approximate' values of the properties could be sufficient. However, due to the extremely sensitive inter-relations between the properties, especially true in various regions, it has been necessary for the simulator to retain as much precision as possible. Very visible inconsistencies in property behavior and consequent coding challenges have emerged, for example, in the liquid region where extremely small changes in specific volume are related to very large changes in pressure, and along the saturated vapor and saturated liquid lines where derivatives of various properties are discontinuous.

Three-dimensional representations of the thermodynamic property relations as defined by the EOS powerfully communicate their interdependent nature. Additional graphics produced in the course of developing the simulator are shown in Figures 2 and 3. Such graphic relationships, while extremely intriguing and instructive, are not typically included in standard thermodynamic textbooks, but are being considered for upper levels of the thermo-game.

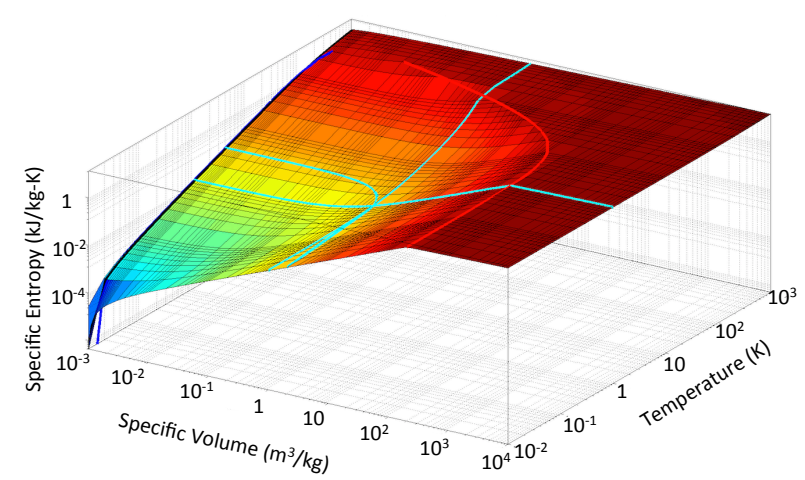

Figure 2: s-v-T surface

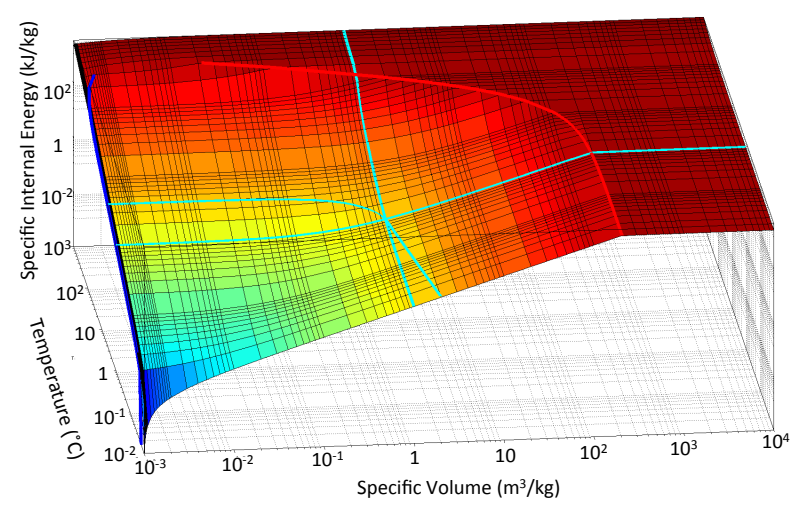

Figure 3: u-T-v surface

Game features: In the initial 'thermo-golf' version of the game, players are able to move a ball from a beginning state to a final state by adding user-chosen increments of energy in the form of work or heat. The amount of energy is selected from a playfully moving scale via a user-click at some precise moment, and the ball subsequently moves along a pre-selected constraint line, such as constant temperature, constant enthalpy, etc. to a final location that is determined by the first law of thermodynamics - that is, an energy balance. In the initial levels of the game, its scope is limited to address closed systems, so that the energy balance determines the value of the final specific internal energy $\left(\mathrm{u}_{2}\right)$ from its initial value $\left(\mathrm{u}_{1}\right)$ and the amount of work performed $\left(\mathrm{W}_{1-2}\right)$ or heat added $\left(\mathrm{Q}_{1-2}\right)$ in the usual manner:

$$
u_{2}-u_{1}=\frac{1}{m}\left(Q_{1-2}-W_{1-2}\right)
$$

A tutorial is provided in the first level of the game, instructing the player how to move around the surface, how to select the various constraint lines, and how to add or subtract a limited number of energy increments in order to reach a well-defined goal. 
The overall look of the 3D environment is patterned after the movie 'Tron,' the goal reflects the style of the light column in 'Wreck-it Ralph,' and a playful music track courses throughout the game.

\section{In Class Use of the Game}

During the fall 2013 semester, Professor Pfotenhauer taught one of the three 60-student-large undergraduate thermodynamics sections at the University of Wisconsin - Madison. As a collaborative effort of the Professors involved, the three sections followed the same syllabus, including homework and exam assessments. In keeping with many introductory courses on engineering thermodynamics, the first two weeks present information to the students regarding a) closed and open systems, b) the first law energy balance relating heat, work, and internal energy, and c) thermodynamic properties.

The game-development team readied the initial version of the game for use in conjunction with a September homework assignment on thermodynamic properties, and students in the PI's section were instructed to play the game as part of their homework assignment. Students in the other two sections, representing the control group, were not introduced to the game but completed the same non-game component of the homework.

Three different assessment methods were used to measure the game's impact as a teaching tool, 1) administering a 30-question subset of the Thermodynamics Concept Inventory ${ }^{10}$, 2) analyzing the digital record of the game play data, and 3) conducting a post-play interview.

The Thermodynamics Concept Inventory (CI), developed by Jacobi, Martin, Mitchell, and Newell ${ }^{10}$ in its full form includes over 480 multiple-choice questions from the topics typically covered in the introductory thermodynamics course for undergraduate mechanical engineering students. A 30-question subset of the inventory, focused on property relations, and slightly modified by replacing 2D graphs with 3D representations of the same ideas was prepared for use along with the game.

Students in all three sections completed the 30-question concept inventory before the topic of thermodynamic properties was introduced, and in the test section before playing the game. The basic features of the game were introduced to students in the test section and they were instructed to play the game as part of their assigned homework activities for the next class. It should be noted that the game portion of the homework assignment did not associate a grade with player performance, rather simply with whether the students played the game or not. Students in all three sections completed the concept inventory a second time following the lectures and homework regarding the topic of thermodynamic properties.

Each student invited to play the game completed a brief registration card identifying their name, gender, whether they had previously taken a thermodynamics course, and whether they play video games more than 2 hours per week. In addition, they received a login / password identification associated with their individual registration card. The registration cards were collected and retained by an external evaluator. Data from the students' interactions with the game included the time-stamped record of each mouse click and their associated choices, such as 
the constraint line, amount of heat or work applied, as well as the beginning and ending locations in the P-v-T space. In that each click (or event) was time stamped, the digital record also included the time between events. Careful analyses of these digital records enabled both an assessment of the students' performance in the game, as well as their interactive responses to the game.

As a third assessment tool, the external evaluator conducted a formative evaluation / survey of the students approximately one month after their interaction with the game. The survey included the 8 questions shown in Table 1 below, and provided the students with a mechanism to numerically rate their interaction with the game, and describe its usefulness as a learning tool.

\section{Assessment Results}

157 students from all three sections of the introductory thermodynamics course participated in the pre-topic CI measurement. Their average score was 39.0\%. 153 students from the same sections participated in the post-topic CI and their average score was $60.4 \%$. The post-topic CI average was statistically the same for students who played the game as for those who did not.

Data gathered directly from the game log revealed a number of interesting features regarding the students' interaction with the game, including some dependence of those interactions on the student demographics. Figure 4 displays a box \& whisker plot reflecting the time spent playing the game per student. The mean value is close to eight minutes, with minimum and maximum values ranging from 2 minutes to 40 minutes respectively. The first and third quartile boundaries are at four and eighteen minutes respectively. The mean value of eight minutes is quite short and indicates that the game did not hold the students' attention very long. Figure 5 displays a graph of the mean time between events in the game. It is a composite of data for all students who played the game, and reflects how much time lapsed between each 'click' or movement within the game. The large grouping of events toward the left end of the graph indicates rapid activity during the game play. This feature would be expected of a game where quick decisions and reflex action would dominate the play. In contrast, a game requiring contemplation or strategy would produce a larger grouping of events toward the right end of the graph. Figure 5 indicates that students interacted with the thermo-golf game using quick decisions and reflex reactions.

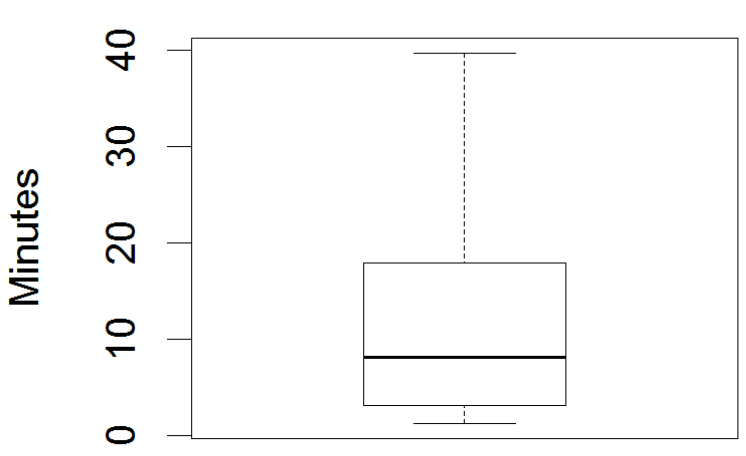

Figure 4. Minutes played per player

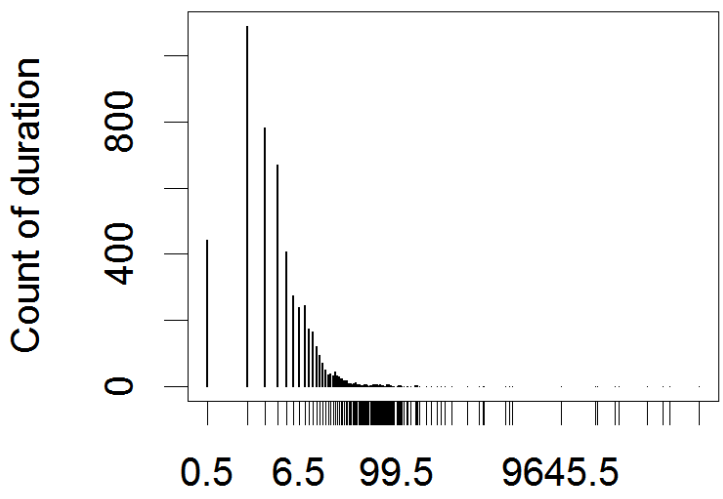

Event Duration

Figure 5. Frequency of game event duration 
By combining the results from the CI measurements with the game data, and focusing only on the first 10 questions of the CI, we observed a lack of correlation between the time spent playing the game, and the improvement in this part of the CI score (Figure 6). The first 10 questions explicitly provided a 3D graphics element along with the question. Clearly, additional time spent interacting with the 3D space did not provide an advantage for learning the related concepts. Furthermore, as reflected in the box \& whisker plot of Figure 7, the CI improvement scores (focused on questions 1-10) for the section of students having played the game (section 1) were statistically no different than those for students who did not play the game, and who therefore had no exposure to the 3D rendering of the PVT space.

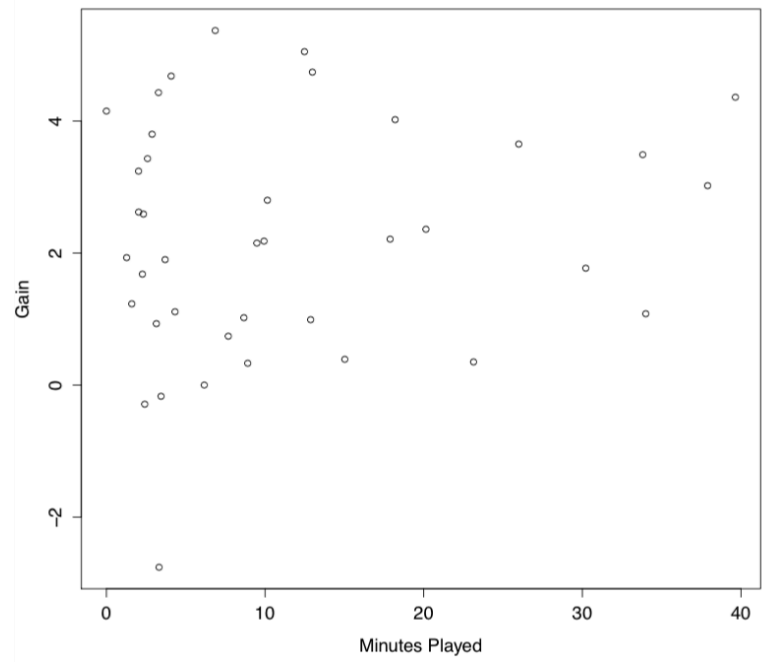

Figure 6: Gain on CI questions 1-10 as a function of minutes played in the game

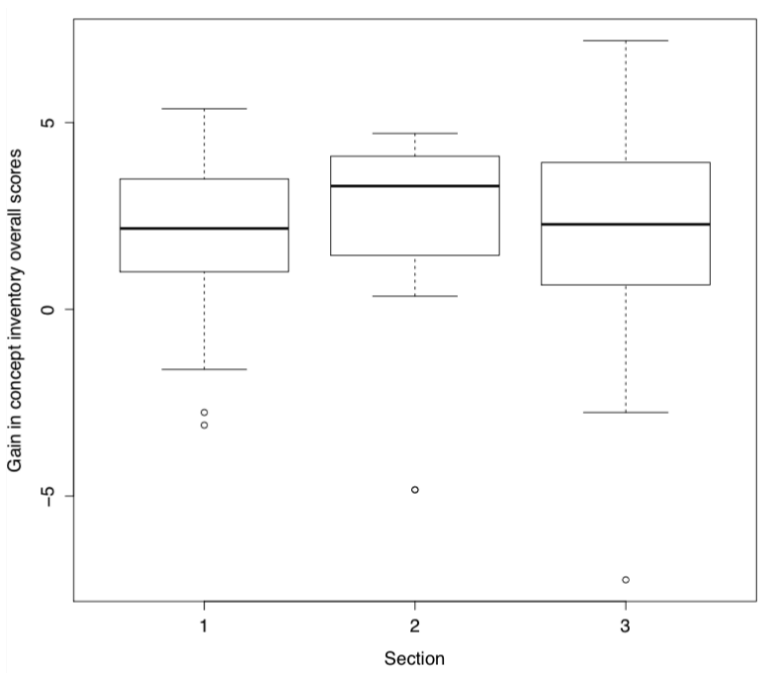

Figure7: Gain on CI questions 1-10 for the three class sections

A sample question from the Concept Inventory that includes a graphics component is shown in Figure 8.

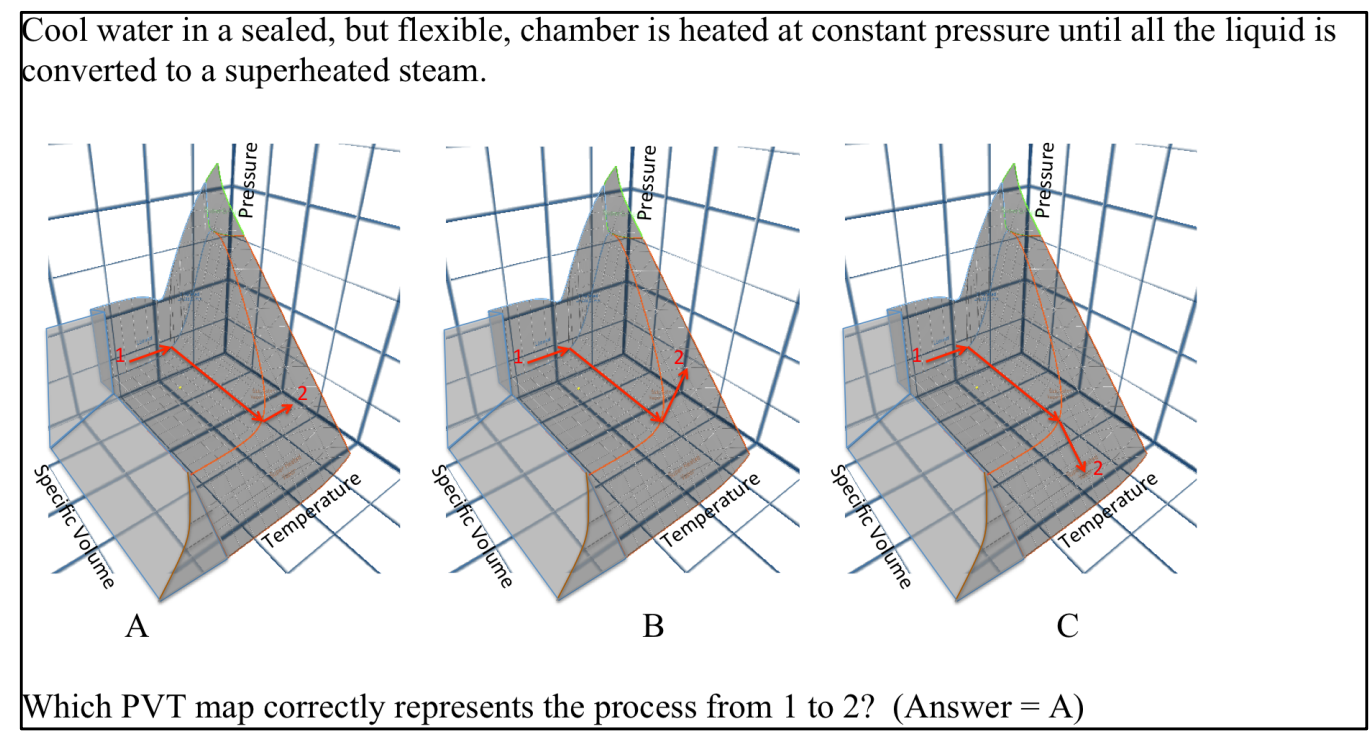

Figure 8 . Concept Inventory question utilizing 3D graphical content 
Reflecting on the short time spent by the students who played the thermo-golf game, and their characteristic 'quick-decision, reflex action' within the game, it may not be surprising that their interaction with the game provided little if any learning advantage for the questions contained within the concept inventory.

\section{Demographic differentiation}

Two demographic distinctions were provided by the students' registration information - gender, and gaming activity. The group of 55 students who played the game included 43 male, and 12 female participants. Roughly $45 \%$ of the male participants were 'gamers' (those who play video games more than 2 hours/week), while only about $10 \%$ of the female participants identified themselves as such.

Data regarding the highest level completed by the students reveals a lower tolerance of the game by the female vs. the male participants. Essentially all of the female participants ended their game play at level one, while a significant portion of the male participants continued on to levels 2,3 , and for one outlier, even to level 4 . The number of game events reflects a similar trend. The mean number of game events generated by the female participants is approximately 35 , while that for the male participants is approximately 90 .

Differentiation in the game data was also apparent across the demographic of gamer and nongamer. Except for 3 outliers amongst the non-gamers, all ended their play after completing level 1. The mean of the completed levels for the gamers is also level 1 , but a significant number of these continued on to levels 2,3, and 4. Although the distinction is not as dramatic, the number of game events generated by the gamers and non-gamers follows the same trend as the number of completed levels. The mean number of events generated by the non-gamers is approximately 50 while the mean number of events generated by the gamers is about 95 .

\section{Interview results}

The external evaluator conducted an interview of 44 students in the test class, approximately one month following their interactions with the game. The questions posed by the survey, along with the dominant $\%$ response are presented in Table 1.

A number of interesting conclusions may be drawn from this data. Of note, the average time students recall playing the game compared to the value provided by the game data is interesting. As is typical of self-reported data, the information is more positive than the externally (game) generated data. According to the answers provided in response to questions 3 and 4, one may claim success for one of the primary objectives of the game: that is to instill a geometric picture of the PVT surface in their minds. However, one of the most significant conclusions drawn from the student survey, as reflected in answers $1,5 \mathrm{c}$, and 6 , is that the students perceived a disconnect between time spent playing the game and their performance (grade) in class. Especially troubling for the game development team was the very cool student response to question 5c. 
Table 1. Interview / Survey Questions and Response

\begin{tabular}{|c|c|c|c|}
\hline \multicolumn{2}{|c|}{ 1. Approximately how long did you play the game? } & \multirow{2}{*}{\begin{tabular}{|c|}
$\begin{array}{c}\% \text { of } \\
\text { respondents }\end{array}$ \\
(average) \\
\end{tabular}} & \multirow{2}{*}{\begin{tabular}{|l|} 
response \\
32.7 minutes \\
\end{tabular}} \\
\hline & & & \\
\hline & $\begin{array}{l}\text { What would have been an incentive for you to play the game } \\
\text { longer? }\end{array}$ & 40 & if game had direct impact on grade \\
\hline & & 29 & resolve some technical issue (game functionality) \\
\hline & & 24 & if more time available or game more engaging \\
\hline & & 7 & no response \\
\hline \multicolumn{4}{|c|}{ 2. At what level did you get 'stuck' and stop playing? } \\
\hline & & (average) & level 2.93 \\
\hline & What caused you to get stuck or stop playing? & & \\
\hline & & 38 & bored or confused \\
\hline & & 26 & some technical feature of game functionality \\
\hline & & 12 & more pressing activities (homework) \\
\hline & & 2 & nothing - game completed \\
\hline \multicolumn{4}{|c|}{ 3. What did you learn from the game about thermodynamics? } \\
\hline & & 45 & some feature of the PVT surface \\
\hline & & 24 & something related to energy and work \\
\hline & & 24 & no new information \\
\hline & & 7 & how $P, v$, and $T$ are related \\
\hline \multicolumn{4}{|c|}{ 4. How did the game relate to what you are learning in this course? } \\
\hline & & 50 & relations to PVT diagram \\
\hline & & 21 & general statement that it related to the class \\
\hline & & 17 & related to phase change and properties \\
\hline & & 7 & related to work, heat, and energy \\
\hline \multicolumn{4}{|c|}{ 5. Indicate your agreement with the following statements (1=strongly disagree, ... 3=neutral, ...5=strongly agree) } \\
\hline a. & I enjoyed playing thermo-game & (average) & 3.07 \\
\hline b. & Playing the game helped me learn thermodynamic concepts & (average) & 3.34 \\
\hline c. & If the game was available to me, I would use it to help me study & (average) & 2.76 \\
\hline d. & I would recommend this game to other engineering students & (average) & 3.02 \\
\hline e. & I would like to play the game again & (average) & 2.85 \\
\hline f. & This game is an effective teaching tool & (average) & 3.34 \\
\hline g. & This game is an efficient teaching tool & (average) & 3.07 \\
\hline \multicolumn{4}{|c|}{ 6. How could this game be used as a teaching tool, if at all? } \\
\hline & & 48 & specific suggestions for content changes \\
\hline & & 14 & connect game play with grades \\
\hline & & 14 & use the game in class \\
\hline & & 12 & use game at alternate point in the class \\
\hline \multicolumn{4}{|c|}{ 7. What would make this game more fun or entertaining? } \\
\hline & & 43 & technical issue related to game functionality \\
\hline & & 31 & no changes needed \\
\hline & & 24 & convert it to a different game type \\
\hline & & 2 & make it more like real life \\
\hline \multicolumn{4}{|c|}{ 8. Any other comments or suggestions? } \\
\hline & & 50 & no response \\
\hline & & 24 & suggestions regarding game functionality \\
\hline & & 17 & general positive comments \\
\hline & & 10 & general negative comments \\
\hline
\end{tabular}

\section{Re-directing the Game Development}

Based on the observations from the game data, and the student survey data, the game development team decided during the latter half of the fall 2013 semester, to dramatically alter the content and style of the thermodynamics game. The revised direction is more precisely aligned with the specific pedagogical objectives for the introductory thermodynamics course, and leads to a variety of professional practice scenarios that had been generated at an earlier stage in the project. 
Examples of the pedagogical objectives include:

- Comparing a given set of pressure and temperature values to the corresponding saturation values in order to identify the associated thermodynamic region (sub-cooled, two-phase, or superheated vapor).

- Recognizing how the properties of specific volume, specific internal energy, specific enthalpy, and specific entropy all scale with quality inside the vapor dome.

- Noticing that in the sub-cooled liquid region, property values at a given temperature and pressure are very similar to the values at the saturated liquid line with the same temperature.

A sample of the envisioned professional practice scenarios include:

- Steam cleaning (as with jewelry): to what pressure should a sealed tank of water be heated to produce the desired quality $(\mathrm{x})$ at the exit of the nozzle?

- Pressure-cooking: what combination of heat (pressure) and time minimizes the amount of energy required to cook xyz?

- Rankine cycle: Optimize the state parameters in a Rankine cycle within given constraints to maximize the cycle efficiency.

In the revised genre of the game, the player uses a throttle that controls the rate at which energy, in the form of either work or heat, is added to the instantaneous state along the various constraint lines (constant pressure, constant temperature, constant internal energy, etc.) in order to reach specific goals on the PVT surface (Figure 9). For example, as part of the introductory tutorial, players are instructed to move to the point of highest temperature. Of the three possible goals to which they could move, one has the highest temperature. Once they arrive at any of the goals, a corresponding message of success or failure appears.

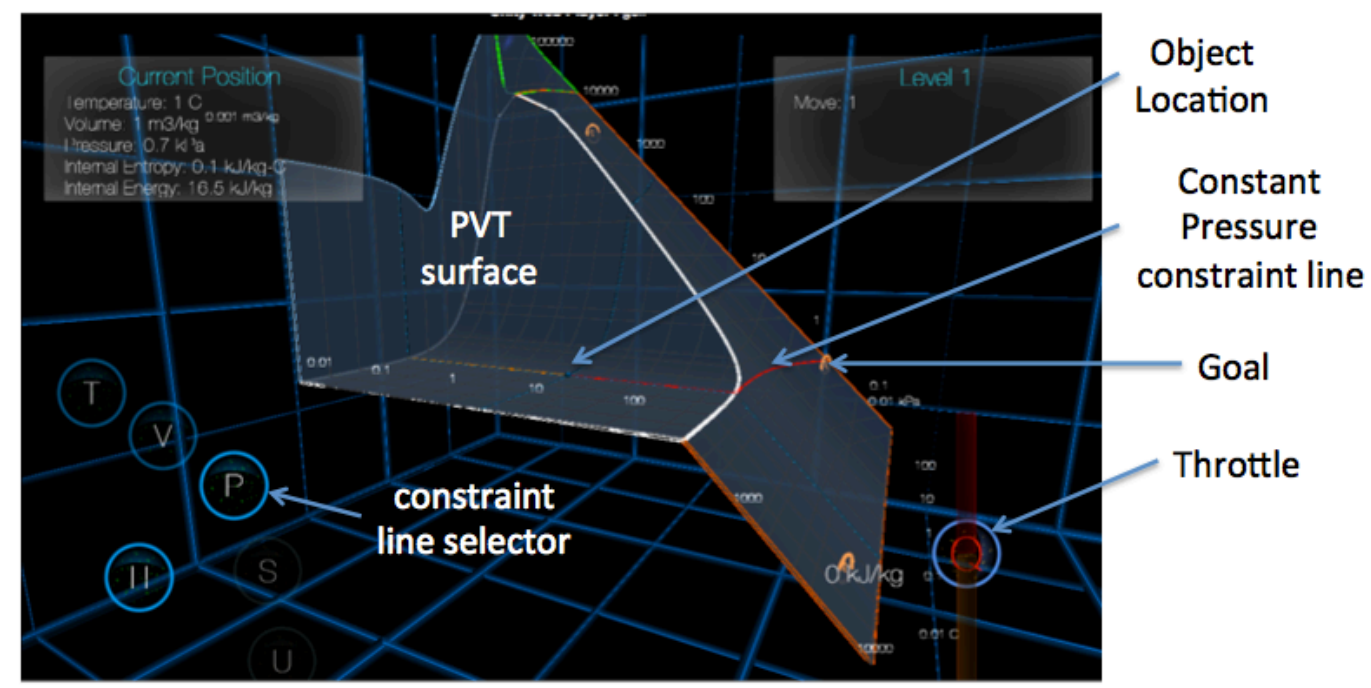

Figure 9: Game view of 3D, P-v-T surface for revised Thermo-game

A close-up of the throttle is shown in Figure 10. Adjusting the position of the slider changes the direction and speed of the object along the constraint line. Moving the slider up from the mid, or zero, position increases the speed in one direction (red) along the constraint line, while moving the slider down from the mid position increases the speed in the opposite direction (orange). The 
throttle is also selectable as either Q (heat) or W (work), and the associated rate of change in the specific internal energy, as defined by the first law of thermodynamics, eq. (1), determine movement along the constraint line. The adjustable slider and selectable constrain lines enable the player to explore the entire surface at a fast or leisurely pace. This feature, along with the continuously updated "Current Position" information box allows the player to observe how the various thermodynamic properties change throughout the different regions and along the defined constraint lines.

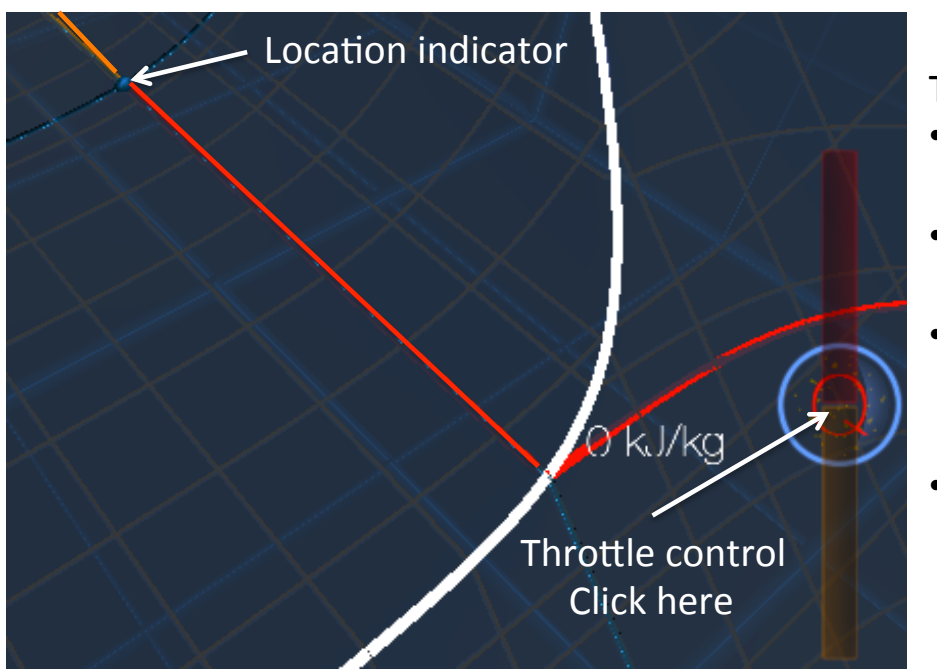

Throttle:

- Shown at mid or zero position

- Shown with energy input selected for $Q$ (heat)

- Click and drag up to increase speed in red direction

- Click and drag down to increase speed in orange direction

Figure 10. Throttle controller in revised game. The speed of movement is controlled by the distance away from the mid, or zero, position of the throttle.

During an in-class play-test of the (unfinished) revised version during the summer 2014 semester, the students identified a variety of functional bugs, but agreed that the game significantly helped them visualize the thermodynamic regions, and could clearly provide a valuable tool for further analyses. One student commented "This is the best visualization graphic I could imagine for the PVT surface."

\section{Formative Feedback Preparations}

One of the primary objectives for the on-line thermodynamics game development is to utilize the digital record of the player moves, both to identify patterns of thought or learning moments, and to provide formative feedback during the game. Various resources from the growing field of Data Mining $^{11}$, Bayesian Knowledge Tracing ${ }^{12}$ (BKT), and Performance Factor Analysis ${ }^{13}$ (PFA) are being readied to develop a concept assessment tool within the thermodynamics game. BKT is expected to be useful for analyzing the data generated by game play because it models student knowledge as a probability rather than a fact. Such an approach may be very appropriate for the risky business of determining student knowledge by analyzing game play data. PFA can also be useful for analyzing the data generated by game play because it takes into account the possibility that students learn not only when they succeed at a task, but also when they fail at the task. Such a feature is true both in real life and in game play, thus the PFA would provide a valuable tool for modeling learning. 
A further goal is to adapt the existing evaluation logic in the assessment tool into a real-time analysis and feedback module that may be used during a live play session. The results of the analysis will be made known to the player as integrated formative feedback, informing the player of observed gaps in their understanding and reinforcing observed proficiencies.

\section{Summary}

Building a game out of a simulation tool can enable an enjoyable learning experience. Subsequently fashioning a simulator-based game into a learning tool represents a notable pedagogical accomplishment. However, as reflected in the results detailed above, engineering undergraduates are still unlikely to utilize such a tool if it does not closely track with the specific pedagogical goals of a defined course syllabus. As Wilson ${ }^{14}$ notes, matching the elements and attributes of a game to the desired learning outcomes is a difficult task.

The thermodynamics game pursued in this project has advanced through simulator development, game enhancement, and educational infusion to its present state where it is being aligned with the specific pedagogical goals of the undergraduate thermodynamics course in mechanical engineering. Along with the envisioned formative feedback tools, soundly based on learning tracing theories, we expect the thermo-game to develop into a very useful tool that will help undergraduate engineering students quickly grasp the key concepts in their introductory thermodynamics course.

\section{References}

1. A.P. Raia, "A Study of the Educational Value of Management Games," The Journal of Business vol. 39 (3), pp. 339-352. (1966)

2. K.J. Chapman and C.L. Sorge "Can a Simulation Help Achieve Course Objectives? An Exploratory Study Investigating Differences Among Instructional Tools," The Journal of Education for Business vol. 74 (4), pp. 225-230. (1999)

3. H.A. Spires, J.P. Rowe, B.W. Mott, and J.C. Lester, "Problem Solving and Game-Based Learning: Effects of Middle Grade Students' Hypothesis Testing Strategies on Learning Outcomes," Journal of Educational Computing Research vol. 44(4), pp. 453-472. (2011).

4. Partnership for 21st Century Skills. [Web site]. Retrieved from http://www. 21stcenturyskills.org (2004).

5. F. Levy \& R. Murnane, The new division of labor: How computers are creating the next job market. Princeton, NJ: Princeton University Press (2004).

6. M.M. McCarthy, "The Role of Games and Simulations to Teach Abstract Concepts of Anarchy, Cooperation, and Conflict in World Politics," The Journal of Political Science Education vol. 10, pp. 400-413. (2014).

7. J.P. van Staalduinen and S. de Freitas, "A Game-Based Learning Framework: Linking Game Design and Learning Outcomes," Chapter 3 of Learning to Play: Exploring the Future of Education with Video Games, Myint Swe Khine, ed., Peter Lang Publishing, Inc. NY, (2011).

8. J.M. Pfotenhauer, D.J. Gagnon, M.J. Litzkow, and C.C. Blakesley, "Designing and Using an On-line Game to Teach Engineering," Proceedings - Frontiers in Education Conference, FIE, v2009, p. W2C/1-5 (2009).

9. W. Wagner and A. Pruss, "The IAPWS Formulation 1995 for the Thermodynamic Properties of Ordinary Water Substance for General and Scientific Use," J. Phys. Chem. Ref. Data, vol. 31(2), pp. 387-535. (2002).

10. A. Jacobi, J. Martin, J. Mitchell, and T. Newell, "Work in progress: a concept inventory for heat transfer" Proceedings - Frontiers in Education Conference, FIE, v2004, p T3F/3-4. (2004). 
11. K.R. Koedinger, E. Brunskill, R.S.J.d Baker, E.A. McLaughlin, and J. Stamper, "New Potentials for DataDriven Intelligent Tutoring System Development and Optimization,” AI Magazine., vol. 34 (3), pp 27-41. (2013).

12. A.T. Corbett, J.R. Anderson, "Knowledge Tracing: Modeling the Acquisition of Procedural Knowledge," UserModeling and User-Adapted Interaction, vol.4, pp. 253-278. (1995).

13. P.I. Pavlik Jr., H. Cen, and K.R. Koedinger, "Performance Factors Analysis - A New Alternative to Knowledge Tracing," Proceedings of the $14^{\text {th }}$ International Conference on Artificial Intelligence in Education, (AIED09) pp. 531-538. Amsterdam, IOS Press. (2009).

14. K.A. Wilson, W.L. Bedwell, et al. "Relationships Between Game Attributes and Learning Outcomes," Simulation \& Gaming, 40 (2), 217-266. (2009). 